Virginia Commonwealth University VCU Scholars Compass

\title{
Genetic and Environmental Structure of DSM-IV Criteria for Antisocial Personality Disorder: A Twin Study
}

Tom Rosenström

Norwegian Institute of Public Health

Eivind Ystrom

Norwegian Institute of Public Health, University of Oslo

Fartein Ask Torvik

Norwegian Institute of Public Health

See next page for additional authors

Follow this and additional works at: http://scholarscompass.vcu.edu/psych_pubs

Part of the Psychiatry and Psychology Commons

(C) Springer Science+Business Media New York 2017

\section{Downloaded from}

http://scholarscompass.vcu.edu/psych_pubs/72

This Article is brought to you for free and open access by the Dept. of Psychiatry at VCU Scholars Compass. It has been accepted for inclusion in Psychiatry Publications by an authorized administrator of VCU Scholars Compass. For more information, please contact libcompass@vcu.edu. 


\section{Authors}

Tom Rosenström, Eivind Ystrom, Fartein Ask Torvik, Nikolai Olavi Czajkowski, Nathan A. Gillespie, Steven H. Aggen, Robert F. Krueger, Kenneth S. Kendler, and Ted Reichborn-Kjennerud 


\title{
Genetic and Environmental Structure of DSM-IV Criteria for Antisocial Personality Disorder: A Twin Study
}

\author{
Tom Rosenström ${ }^{1}$ • Eivind Ystrom $^{1,2,3} \cdot$ Fartein Ask Torvik $^{1}$. \\ Nikolai Olavi Czajkowski ${ }^{1,2} \cdot$ Nathan A. Gillespie $^{4} \cdot$ Steven H. Aggen $^{4}$. \\ Robert F. Krueger ${ }^{5}$ Kenneth S. Kendler ${ }^{4,6,7}$ - Ted Reichborn-Kjennerud ${ }^{1,8}$
}

Received: 9 August 2016/ Accepted: 23 December 2016 / Published online: 21 January 2017

(C) Springer Science+Business Media New York 2017

\begin{abstract}
Results from previous studies on DSM-IV and DSM-5 Antisocial Personality Disorder (ASPD) have suggested that the construct is etiologically multidimensional. To our knowledge, however, the structure of genetic and environmental influences in ASPD has not been examined using an appropriate range of biometric models and diagnostic interviews. The 7 ASPD criteria (section A) were assessed in a population-based sample of 2794 Norwegian twins by a structured interview for DSM-IV personality disorders. Exploratory analyses were conducted at the
\end{abstract}

Edited by Michael Lyons.

Electronic supplementary material The online version of this article (doi:10.1007/s10519-016-9833-z) contains supplementary material, which is available to authorized users.

Tom Rosenström

tom.rosenstrom@helsinki.fi

1 Department of Mental Disorders, Norwegian Institute of Public Health, Oslo, Norway

2 Department of Psychology, University of Oslo, Oslo, Norway

3 PharmacoEpidemiology and Drug Safety Research Group, School of Pharmacy, University of Oslo, Oslo, Norway

4 Department of Psychiatry, Virginia Institute for Psychiatric and Behavioral Genetics, Virginia Commonwealth University, Richmond, VA, USA

5 Department of Psychology, University of Minnesota, Minneapolis, USA

6 Deparment of Human and Molecular Genetics, Virginia Commonwealth University, Richmond, VA, USA

7 Department of Psychiatry, Virginia Commonwealth University, Richmond, VA, USA

8 Institute of Clinical Medicine, University of Oslo, Oslo, Norway phenotypic level. Multivariate biometric models, including both independent and common pathways, were compared. A single phenotypic factor was found, and the best-fitting biometric model was a single-factor common pathway model, with common-factor heritability of $51 \%$ (95\% CI 40-67\%). In other words, both genetic and environmental correlations between the ASPD criteria could be accounted for by a single common latent variable. The findings support the validity of ASPD as a unidimensional diagnostic construct.

Keywords Unidimensionality · Common pathway · Multivariate biometric model · Psychometrics · Diagnostics

\section{Introduction}

Understanding the etiology of antisocial behavior and criminality is important, given their high societal costs. Twin and adoption studies have estimated that genetic influences account for roughly $40 \%$ of the variance in antisocial behavior across assessment methods (Rhee and Waldman 2002), but findings from molecular genetic studies have been inconsistent and have failed to replicate (Kendler 2006; Tielbeek et al. 2012; Ficks and Waldman 2014; Salvatore et al. 2015; Pappa et al. 2016). In addition to statistical power issues and potential gene-environment interactions, another possible explanation for mixed findings is that the assessed phenotypes, such as the DSM-IV Antisocial Personality Disorder (ASPD), reflect multiple etiologically distinct factors. This is plausible given that ASPD diagnosis is based on seven different criteria which may or may not reflect a unidimensional liability factor [an eighth criterion, childhood conduct disorder, is required for diagnosis, but often studied separately (Kendler et al. 2012, 
2013; American Psychiatric Association 2013; Venables et al. 2014; Derefinko and Widiger 2016)].

Developmental studies of antisocial behavior suggest that it is more heritable when combined with callousunemotional traits than when these traits are not present; among incarcerated adults, this trait combination is called "psychopathy" (Viding and McCrory 2012). Psychopathy is also frequently modeled as a four-dimensional construct, involving variation along interpersonal, affective, lifestyle, and "antisocial behavior" dimensions (Neumann et al. 2015). What may be confusing to many is that the content of the seven ASPD criteria distribute to all these dimensions instead of just the "antisocial" dimension.

The first ASPD criterion assesses failure to conform to social norms, "as indicated by repeatedly performing acts that are grounds for arrest" (could fit the "antisocial" psychopathy dimension). The second criterion assesses deceitful behaviors (interpersonal dimension). The third criterion assesses impulsivity or failure to plan ahead (lifestyle dimension). The fourth criterion assesses irritability and aggressiveness "indicated by repeated physical fights or assaults" (antisocial dimension). The fifth criterion assesses reckless disregard for safety of self or others (lifestyle dimension). The sixth criterion assesses consistent irresponsibility regarding work behavior or financial obligations (lifestyle dimension), and the seventh criterion assesses lack of remorse, "as indicated by being indifferent to or rationalizing having hurt, mistreated, or stolen from other" (affective dimension). The partial correlations between the psychopathy dimensions and sum of ASPD criteria directly reflect the above content analysis, with the interpersonal dimension (represented by one ASPD criterion) being least correlated with ASPD sum score and the lifestyle dimension (represented by 3 criteria) most correlated with the sum score (e.g., Table 1 in Coid and Ullrich 2010).

While the four psychopathy dimensions are correlated and consistent with "a fundamental link between antisociality and other features of psychopathy" (Neumann et al. 2015), the apparent dispersal of ASPD content across such multiple dimensions rises questions regarding homogeneity of the ASPD construct. Yet, in factor analyses of all the criteria of all or multiple DSM-IV personality disorders, ASPD has been among the disorders that most consistently load onto a single factor (Blais and Norman 1997; Warren and South 2009; Huprich et al. 2010). That is at the "phenotypic" level, referring to study of unrelated individuals. Family studies provide a unique opportunity to further understand the population variation in characteristic patterns of both normal and disordered behavior, or personality (Franić et al. 2013; Livesley 2005).

To our knowledge, only one genetically informative study on the dimensional (factor) structure of ASPD criteria has been published (Kendler et al. 2012). Kendler et al. (2012) found evidence for two correlated phenotypic factors, dubbed "aggressive-disregard" (ASPD criteria \#1, \#4, and \#5) and "disinhibition" (criteria \#2, \#3, \#6, and \#7). Multivariate twin modeling then identified two genetic factors underlying the phenotypic factors (though criterion \#7 was not well-represented) and an additional environmental factor, along with criteria-specific genetic and environmental effects. This led the authors to conclude that from a genetic perspective, the DSM-IV criteria for ASPD do not reflect a single dimension of liability, but instead two dimensions of genetic risk reflecting aggressive-disregard and disinhibition influence ASPD. However, they tested only a limited number of biometric models, and assessed the ASPD criteria by self-report questionnaire items that were mapped onto the DSM-IV ASPD criteria.

The previously tested set of biometric models was limited in the sense that it contained only "independent pathway" models and no "common pathway" models (Neale and Maes 2002; Franić et al. 2013; Livesley 2005; Markon and Krueger 2004). This means that genetic and environmental factors were assumed to independently influence the ASPD criteria, even though there is some evidence that "the phenotype of antisocial behavior is much more than a sum of the genetic and environmental parts" (Derefinko and Widiger 2016; Hyde et al. 2016; Viding and McCrory 2012). A common pathway model instead assumes that genes and environment influence an intermediate phenotype (latent factor) that can further influence the criteria, and is a frequently considered alternative for the independent pathway model. In addition to studying common pathway models, it is of interest to extend the biometric results on self-reported ASPD criteria to widely used interview criteria because assessment method moderates the estimated heritability of antisocial behavior (Rhee and Waldman 2002). In this paper, we therefore: (1) replicate the previous multivariate biometric study on the genetic and environmental structure of DSM-IV ASPD criteria using data from structured interviews instead of self-report questionnaire items, and (2) extend the analyses by applying previously unstudied common pathway biometric models in addition to the independent pathway models.

\section{Methods}

\section{Sample}

Participants in the present study were recruited from the Norwegian Institute of Health Twin Panel, a populationbased sample of Norwegian twins (Harris et al. 2002). Psychiatric Axis I and II disorders were assessed at interview in 2801 twins (43.5\% of those who were eligible) between 
the years 1999 and 2004. Their mean age was 28.2 years and age range 19-36. Zygosity was determined by a combination of questionnaire items and genotyping, resulting in a less than $1 \%$ miss-classification rate, which is unlikely to substantially bias results (Neale 2003). The sample has been used in many previous investigations (Kendler et al. 2008; Tambs et al. 2009; Torgersen et al. 2008, 2012; ReichbornKjennerud et al. 2013). It included 225 monozygotic (MZ) male-twin pairs with data on ASPD criteria (with 5 pairs lacking the other member), 120 dizygotic (DZ) male-twin pairs (including 3 part pairs), $453 \mathrm{MZ}$ female-twin pairs (4 partial pairs), $267 \mathrm{DZ}$ female-twin pairs (8 partial pairs), and 343 pairs of DZ opposite-sex twins (2 partial pairs); a total of 2794 individuals and 1408 (full or partial) twin pairs. To assess sex effects and to compare with a previous study on same-sex twins (Kendler et al. 2012), we also studied the full pairs of same-sex twins (total $n=2090$; twin-pair $n=1045$ ). Approval was received from The Norwegian Data Inspectorate and the Regional Committee for Medical and Health Research Ethics, and written informed consent was obtained from all participants after a complete description of the study.

\section{Measures}

Personality disorders were assessed using a Norwegian version of the Structured Interview for DSM-IV Personality (Pfohl et al. 1995). The method was initially developed in 1983, and has been used in a number of studies in many countries including Norway (Torgersen et al. 2001; Helgeland et al. 2005). It is a comprehensive semi-structured interview of all DSM-IV Axis II diagnoses, rating the specific DSM-IV criteria according to following guidelines: $0=$ not present or limited to rare isolated examples; $1=$ subthreshold (some evidence of the trait, but not sufficiently pervasive for the criterion to be considered present); $2=$ present (criterion clearly present for most of the time during last 5 years); $3=$ strongly present (associated with subjective distress or some impairment in social or occupational functioning or intimate relationships). The criteria were modeled based on an inferred ordered continuous threshold liability model of the endorsed ordinal category frequencies (e.g. polychoric correlations); to lessen the impact of empty cell conditions, the ordinal classes 2 and 3 were collapsed into a single class.

Most of the interviewers were psychology students in their final part of training or experienced psychiatric nurses. They were trained by professionals (1 psychiatrist and 2 psychologists) who had extensive previous experience with the instrument, and they were closely followed up individually during the entire data collection period. Most of the interviews were conducted face to face, but for practical reasons, $231(8.3 \%)$ were obtained by telephone. Each twin in a pair was interviewed by a different interviewer. Interrater reliability was assessed based on 2 raters' scoring of 70 audiotaped interviews: intra-class correlation of 0.91 for the number of endorsed ASPD criteria at the subthreshold level has been reported (Torgersen et al. 2008).

\section{Statistical analyses}

We first investigated the number of phenotypic factors for the 7 ASPD criteria to map the manifest structure of the criteria, and then proceeded to carry out biometric analysis. Sex differences have been studied a lot for antisocial behavior (Rhee and Waldman 2002), and while we lacked power to adequately test for sex-limited genetic effects (Neale et al. 2006; Torgersen et al. 2008; Reichborn-Kjennerud et al. 2015), we studied structural invariance of ASPD with respect to sex both phenotypically and biometrically, as explained below. This was done using the full pairs of same-sex twins, and when no sex differences were found, the models were estimated for the entire sample.

Phenotypic Analyses First, an omnibus test of sex differences in the phenotypic correlations was conducted using a random permutation test (2000 permutations) on the Frobenius norm of the male-female difference in correlation matrices (i.e., their Euclidian distance) (Good 2005). Permuting the male-female status leaves the twin-dependencies unchanged in the permutation/comparison distribution, and therefore the test is not biased by the non-independence of twins. A polychoric approach for ordinal-item endorsement assumes (in this case) that latent liabilities of individuals to endorse a criterion are normally distributed and an individual endorses the criterion in category "1" when his or her liability exceeds the lower estimated threshold but is below the upper estimated threshold, and endorses it in category " 2 " when the latent liability exceeds the upper threshold (the lower bound of the first category is $-\infty$ and the upper bound of last the category is $+\infty$ ). Different criteria can have different thresholds, and men and women can differ in all these thresholds (excluding the "infinity bounds").

Exploratory Factor Analysis (EFA) is a traditional method for investigating the covariance structure among multiple assessment items (e.g. ASPD criteria) to find evidence on shared liability factors (Lawley and Maxwell 1971). The aim is to identify the minimum number of latent factors that can account for the shared covariance among the items. We conducted EFAs using Mplus version 7.31 using the mean- and variance-adjusted weightedleast squares estimator and the complex-sample option (sandwich estimator) to account for the non-independence between twins (Asparouhov 2005; Kendler et al. 2012). Other computations than phenotypic factor analyses were carried out in R software version 3.2.2. 
Because the frequently used likelihood-ratio test for factor number can be biased towards extracting too many factors (Hayashi et al. 2007), we used the Parallel Analysis test applied to polychoric correlations to identify the number of factors in our data (Horn 1965; Humphreys and Montanelli Jr 1975). In parallel analysis test, one generates the same number of uncorrelated observations as in the real data to gauge the extent that mere sampling variability inflates observed correlations, or the eigenvalues reflecting them. The usual Scree plot is then compared to the synthetic nullcorrelation Scree plot to avoid over-extracting factors from sampling variability/noise. Instead of computing effective degrees of freedom, which is a number between the number of twin pairs and the number of twins, we simply show that both the boundary values lead to a same conclusion herein (Jones 2011). In case of disagreement, we also ran a confirmatory factor analysis to verify that the model implied by our EFA supersedes the previously found model, at least in our data. This was done using the "MLR" estimator of Mplus (robust maximum likelihood for non-normal and dependent observations) that allows likelihood-based inference and the information criteria described below.

Differential Item Functioning (DIF) has been previously reported for 3 of the ASPD criteria (Jane et al. 2007). DIF with respect to sex means that one sex endorses a specific criterion differently from the other sex despite adjusting for possible differences in the overall (or 'latent') ASPD between the sexes (Penfield and Camilli 2007). We tested DIF using "lordif" $\mathrm{R}$ package, which is an automated procedure for flagging ordinal items with DIF [with options: significance level 0.01, Chi-squared detection criterion, and minimum cell count of 4 (Choi et al. 2011)].

Biometric Analyses Although not a novel idea (Heath et al. 1989; Kendler et al. 1992), researchers have been increasingly interested in the possibility of clarifying diagnostic constructs using samples of twins (Kendler et al. 2008, 2012, 2013; Reichborn-Kjennerud et al. 2013; Franić et al. 2013; Livesley 2005; Jang et al. 2002; Johnson and Krueger 2004). In traditional phenotypic EFA approaches, there is no way to differentiate the contribution of genetic versus environmental effects in the covariance between the diagnostic items, and therefore no way to know whether they conflict and thus confound the structural inferences based on EFA (Franić et al. 2013). Because MZ twins share roughly $100 \%$ of their segregating genes and DZ twins only on average $50 \%$, their respective criteria correlations can be used to partition the covariance structure of the criteria into distinct genetic and environmental sources of variation (Neale and Maes 2002; Plomin et al. 2012). In the classic twin model, the covariance is partitioned into Additive genetic effects (A), Common or shared environmental exposures that make twins similar $(\mathrm{C})$, and non-shared Environmental effects (E), which comprise all influences making twins different, including measurement error (Neale and Maes 2002; Plomin et al. 2012). When an EFAtype model is applied to these distinct sources of betweenperson variation in diagnostic criteria, we will refer to it as "biometric factor analysis".

In a "common pathway model", the diagnostic criteria reflect one or multiple latent factors each possibly influenced by A, C, or E (Neale and Maes 2002; Franić et al. 2013). A or C contributions can be negligible and sometimes are dropped from the model, but $\mathrm{E}$ is always included because it theoretically includes ubiquitous measurement errors. Figure 1a illustrates an example of a singlefactor common pathway model with four observed criteria. According to the present notation, this is a one-factor model, with only A-E part of the A-C-E partitioning available in twin studies, denoted here by " $1-\mathrm{A}-\mathrm{E}$ " for the factor part and by "a-e" for the specific-effects part. These models can be extended to include two, three or any number of latent factors each influenced by $\mathrm{A}, \mathrm{C}$ and $\mathrm{E}$. The biometric factor analysis model can also be specified as an "independent pathway model" wherein each of the criteria is directly influenced by one or multiple genetic and environmental factors. Figure $1 \mathrm{~b}$ shows a model with only one set of $\mathrm{A}$ and $\mathrm{E}$ factors. Independent pathway models thus can estimate separate latent factors for each of the modeled A, $\mathrm{C}$, and $\mathrm{E}$ covariance components, whereas common pathway models estimate A, C, and E components separately for each of the latent factors. Panels c and d in Fig. 1 exemplify further possible models.

All the previously studied independent pathway models (Kendler et al. 2012) and their corresponding common pathway biometric models were estimated using the "raw data" (full-information maximum likelihood) option of the Open Mx software for structural equation modeling [this makes twin pairs with a missing member usable, implying a total of 2816 informative twins (Boker et al. 2011)]. Confidence intervals (CI) are 95\% likelihood-based intervals (Neale and Miller 1997).

The importance of explicitly comparing the commonand independent pathway models is exemplified by recent studies on borderline personality disorder, a diagnosis closely related to ASPD (Torgersen et al. 2008; ReichbornKjennerud et al. 2013, 2015). A common-pathway model rather than any of the studied independent-pathway models was found to be the "best" description for the borderline personality criteria (Reichborn-Kjennerud et al. 2013). When comparing models, we used Bayesian Information Criterion (BIC) which has been both applied previously for ASPD and shown to perform well for the models of this type in general (Kass and Raftery 1995; Kendler et al. 2012; Markon and Krueger 2004). BIC is a rough approximation for minus twice the logarithm of Bayes Factor, with a difference of 10 or more being considered as very strong 


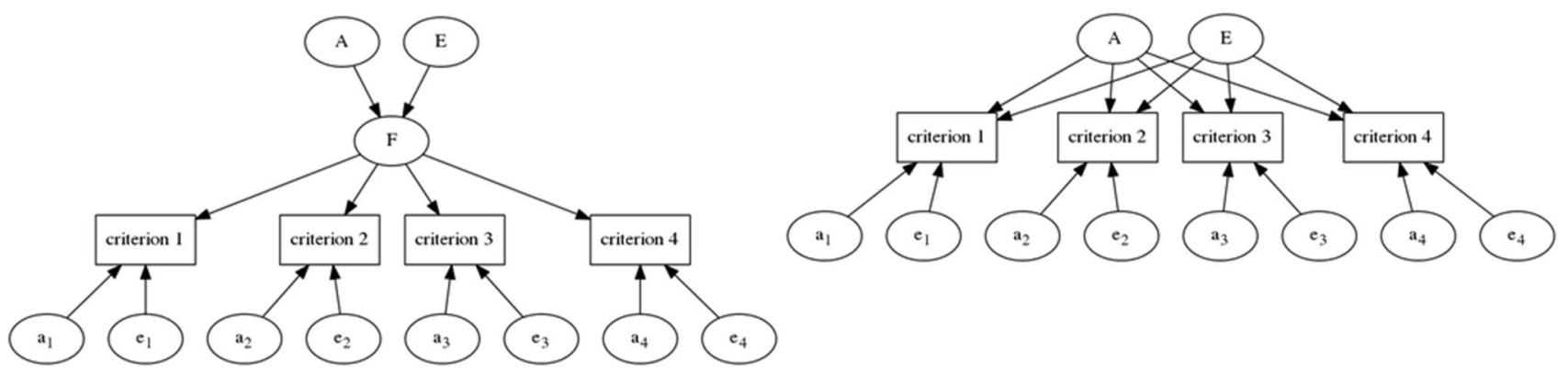

C

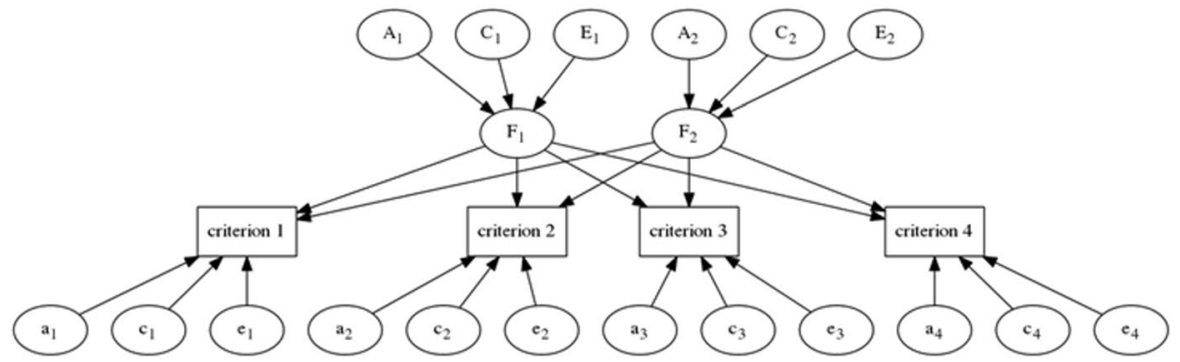

d

2-ACE factor common pathway model independent pathway model

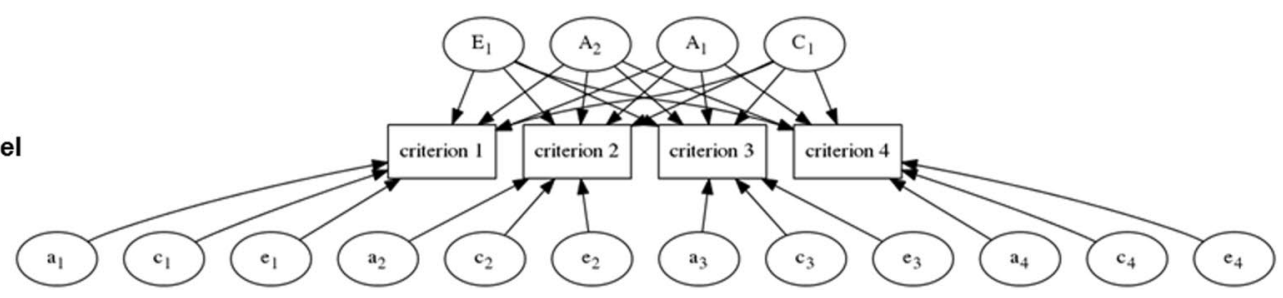

Fig. 1 Examples of alternative common and independent pathway biometric models. a A path diagram of a common pathway biometric factor analysis model without shared environmental effects is shown for 4 criteria for illustration. The common factor $\mathrm{F}$ is partitioned into heritable variance $\mathrm{A}$ and environmental variance $\mathrm{E}$, both of which similarly drive the individual psychiatric criteria. The criteria can have both heritable and environmental specific variances too (lowercase letters). Unobserved variables (ellipses) have unit variance, but may have distinct loading weights (associated with arrows) onto the observed variables (rectangles). b A path diagram of an analogous independent-pathway biometric factor analysis model (a one-genetic factor, zero-shared-environmental factor, and one-non-shared environmental factor model, i.e. "1A-0C-1E" model, or "1A-1E" model. c A path diagram for a biometric model with two common pathways for the $\mathrm{A}, \mathrm{C}$, and $\mathrm{E}$ variance components. $\mathbf{d} \mathrm{A}$ path diagram for a biometric model with two independent genetic pathways, one non-shared environmental, and one shared environmental pathway evidence for the model with the lowest BIC, and anything less than 2 barely worth mentioning (Kass and Raftery 1995). As a test of robustness for the selected information criterion, we compared results for those obtained with another well-performing measure, Sample-size Adjusted BIC [SABIC (Sclove 1987; Markon and Krueger 2004; Nylund et al. 2007)].

As in a previous study on ASPD (Kendler et al. 2012), we assessed overall invariance of factor structure over the sexes in a baseline biometric model by constraining its (non-threshold) parameters across the sexes using the same-sex twins only, and if evidence is found for invariance, compared the rest of the models using full data. However, it is possible that the best-fitting biometric model has more statistical power to detect DIF by sex than baseline models or omnibus tests. A connection between certain factor analysis models and classic DIF exists (Muthén 1989; Muthén et al. 1991; MacIntosh and Hashim 2003), allowing us to evaluate DIF also in the context of the biometric models by comparing models that impose different constraints. We explain this more thoroughly in the supplementary material, and only briefly outline the key points here: (1) if one constrains the factor loadings across the sexes, the modeled criteria are rendered equally sensitive to changes in the latent factor for both the sexes (equal "discriminability" for both sexes in DIF parlance). (2) If one also constrains the residual variances of the factor model, the sensitivity of the criteria for the latent trait has equal mode of inheritance (equal biometric structure) across the sexes. (3) Constraining the latent factor's ACE partitioning 
further implies equal mode of inheritance for the latent scores across the sexes. (4) If one further constrains the criteria thresholds except for a constant translation of all the thresholds for the other sex (cf. Supplementary Figure S1), there is almost no evidence for DIF, only an overall sex difference in the factor scores (see supplementary material for the exact interpretation and a more explicit explanation of biometric DIFs).

\section{Results}

\section{Phenotypic analysis}

Table 1 displays the category endorsement rates for each of the ASPD symptoms. Excluding the conduct-disorder criterion, only 11 participants $(0.4 \%)$ had fully endorsed three or more symptoms as suggested in the diagnostic algorithms, whereas altogether $76(2.7 \%)$ had at least three subthreshold endorsements, $113(4.0 \%)$ had at least one full endorsement, and 517 (18.5\%) had at least one sub-threshold endorsement. Furthermore, 109 (3.9\%) fulfilled the conduct disorder, with 426 (15.2\%) having a sub-threshold endorsement. No sex differences were found in the phenotypic (polychoric) correlations $(\Delta$ Frobenius norm $=1.945$, $p=0.209$ ), but on average, men endorsed 0.10 ASPD criteria (0.50 if including sub-threshold level) and women only 0.03 criteria ( 0.18 if including sub-threshold endorsements). Based on contingency table analyses, each individual criterion had a significant sex difference $\left[\chi^{2}(1)>3.858\right.$, $\mathrm{p}<0.05$ for all], with criterion 6 ("irresponsibility") being borderline significant only $\left[\chi^{2}(1)=3.637, p=0.057\right]$.

The parallel-analysis testing for the number of factors indicated that a single factor was adequate (Fig. 2; for the factor loadings, see Table 1, EFA column). When comparing confirmatory models, the single factor solution (BIC=6777.7; SABIC $=6711.0)$ outperformed the previously reported two-factor solution with criteria \#1, \#4, and \#5 loading on the first factor and the rest on the second factor $(\mathrm{BIC}=6782.9$; $\mathrm{SABIC}=6713.0)$. On the phenotypic level, the automated "lordif" procedure applied to the same-sex twin data flagged just one criterion for DIF, the criterion 6 ("irresponsibility"; all $\chi^{2}$ indices had $p<0.001$ ): given the same total/latent ASPD, women were more likely to endorse the criterion 6 compared to men. Few women had high levels of ASPD, however, and the detected DIF had a negligible effect on the estimated latent ASPD scores ( $<0.01$ s.d. in mean and median difference). In addition, DIF by zygosity can be concern for factor studies (Neale et al. 2005), but also therein the "lordif" procedure flagged just one criterion ("Recklessness"; $p=0.002$ for uniform DIF, $p=0.272$ for non-uniform).

\section{Comparison of biometric models}

As done previously, we tested in same-sex twins whether constraining all parameters except the thresholds across men and women improved the fit of $1 \mathrm{~A}-1 \mathrm{C}-1 \mathrm{E}$ factor independent pathway and 1-ACE factor common pathway biometric models. In both the cases (supplementary Table S3),
Table 1 Criteria endorsement and 1-factor exploratory factor analysis (EFA) loadings

\begin{tabular}{|c|c|c|c|c|c|}
\hline ASPD criterion & Missing & Not endorsed & Sub-threshold & Endorsed & EFA \\
\hline \multicolumn{6}{|l|}{ Men } \\
\hline 1. Not conforming & 2 & 872 & 116 & 34 & 0.789 \\
\hline 2. Deceitfulness & 2 & 916 & 102 & 4 & 0.617 \\
\hline 3. Impulsivity, or failure to plan & 3 & 976 & 33 & 12 & 0.725 \\
\hline 4. Irritability/repeated fights & 2 & 1006 & 8 & 8 & 0.818 \\
\hline 5. Reckless disregard & 3 & 935 & 70 & 16 & 0.608 \\
\hline 6. Irresponsibility & 2 & 946 & 63 & 13 & 0.752 \\
\hline 7. Lack of remorse & 3 & 983 & 27 & 11 & 0.915 \\
\hline \multicolumn{6}{|l|}{ Women } \\
\hline 1. Not conforming & 6 & 1693 & 58 & 20 & - \\
\hline 2. Deceitfulness & 6 & 1698 & 69 & 4 & - \\
\hline 3. Impulsivity, or failure to plan & 11 & 1717 & 40 & 9 & - \\
\hline 4. Irritability/repeated fights & 6 & 1761 & 3 & 7 & - \\
\hline 5. Reckless disregard & 11 & 1737 & 22 & 7 & - \\
\hline 6. Irresponsibility & 6 & 1699 & 58 & 14 & - \\
\hline 7. Lack of remorse & 11 & 1752 & 11 & 3 & - \\
\hline
\end{tabular}

The factor loadings are for the full data, including men and women, because no sex differences were detected in the criteria correlation matrix 

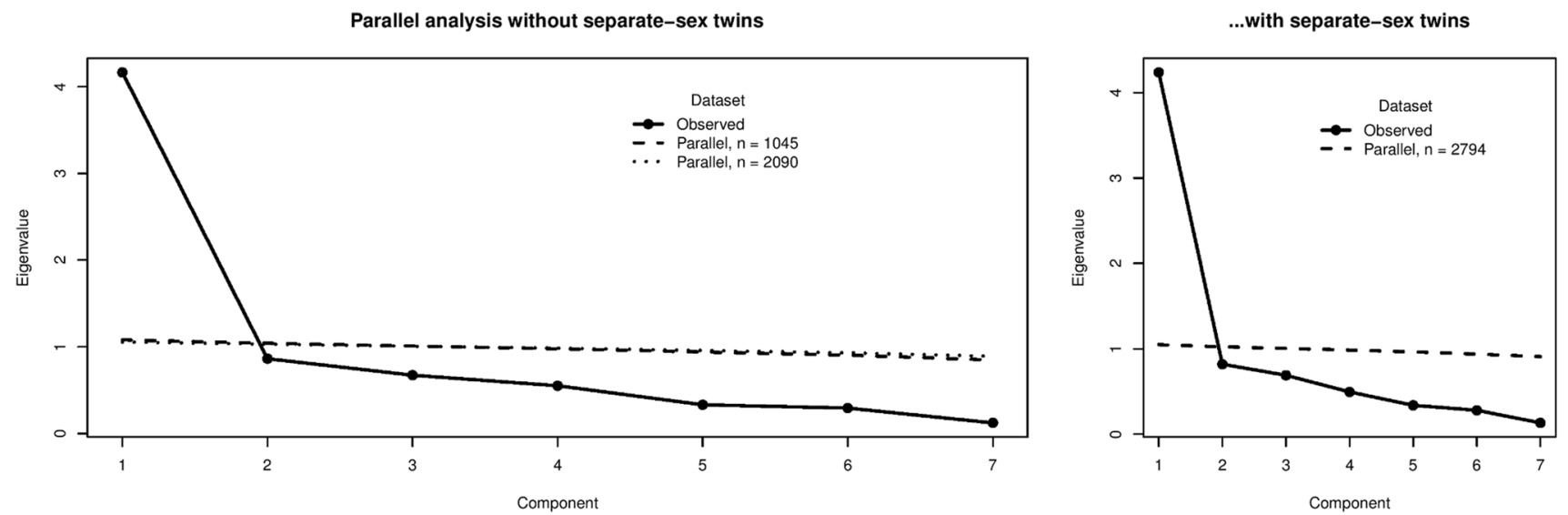

Fig. 2 Scree plot and parallel analysis test for ASPD criteria. First panel The solid line shows the eigenvalues of the weighted-least squares mean- and variance-adjusted polychoric correlation matrix, whereas the dashed (simulated sample size $\mathrm{n}=1045$ ) and the dotted (simulated sample size $n=2090$ ) lines indicate 5th percentile values across 1000 replications in parallel analyses using uncorrelated criteria. Scree-plotted observed eigenvalues above the parallel-analyses

the independent and common pathway models with sex effects absent provided better fits to the data.

In the full data with opposite-sex twins included, the best fitting independent pathway model according to both BIC and SABIC was the Model III that had one genetic factor and one factor for the non-shared environment (Table 2). Among the common pathway models, Model IV had the most parsimonious fit. Importantly, the best common pathway model had a better fit than the best independent pathway model according to BIC, but not according to SABIC. In the same-sex twin data, however, the common pathway model outperformed all independent pathway models also according to SABIC (Table S3). When the conduct-disorder criterion was included, the conclusion was again the same according to BIC, but SABIC picked out yet another model (Table S3). Thus, the common pathway Model IV was the most robust 'best' fit model among those examined. We used this model to evaluate possible biometric DIF in the same-sex twin data.

The common-factor Model IV was estimated with all the parameters set free across the sexes $(\mathrm{BIC}=$ -97011.4; SABIC =4778.3; df=14,592), by constraining only factor loadings to be equal across the sexes $(\Delta \mathrm{BIC}=-37.8 ; \Delta \mathrm{SABIC}=-22.6 ; \Delta \mathrm{df}=6)$, by constraining both factor loadings and specific/residual variances to be equal across the sexes $(\triangle \mathrm{BIC}=-78.4$; $\triangle \mathrm{SABIC}=-67.4 ; \Delta \mathrm{df}=13)$, by fixing all the parameters across the sexes, except for the ordinal criterion thresholds $(\triangle \mathrm{BIC}=-93.3 ; \Delta \mathrm{SABIC}=-75.9 ; \Delta \mathrm{df}=15)$, and by constraining all parameters to be equal across sex except for a uniform scalar translation in men's criteria lines represent structure (i.e., factors) over and above sampling variance. The two parallel analyses lines simply indicate that both perfect correlation (minimum information) and no correlation (maximum information) between the twins would nevertheless lead to the same conclusion. Second panel Same as the first panel, but with using the full data, including separate-sex dizygotic twins. The same conclusion holds for both the panels

endorsement liabilities relative to women (i.e., no other differences but overall higher endorsements in men). This latter model provided the best fit to the data $(\triangle \mathrm{BIC}=$ $-137.1, \triangle \mathrm{SABIC}=-78.4$ ). Thus, we did not detect any omnibus sex differences in the biometric measurement models.

\section{The best fit biometric model}

Although models with multiple common pathways were tested (Table 2 and Table S3), the best-fit model had only one factor with $51 \%$ heritability $(\mathrm{CI}=40-67 \%)$ and $49 \%$ contribution from non-shared environment $(\mathrm{CI}=33-65 \%)$, with no shared environmental effects. Figure 3 shows a path diagram and parameter estimates for this model. Clearly the genetic and non-shared environmental effects conform to the same factor we observed in the phenotypic analysis (Table 1). However, statistically significant criterion-specific genetic effects emerge in the biometric analysis $\left[\chi^{2}(7\right.$ d.f. $)=20.6, p=0.004$ in likelihood-ratio test], showing that the unique variances of EFA contain more than just measurement errors. Based on the estimated thresholds' scalar shift men were on average 0.48 standard deviations $(\mathrm{CI}=0.39-0.58)$ higher on the liability to endorse any of the ASPD criteria compared to women. The best-fit model directly implies the extent of genetic and environmental contributions per criterion, and what proportion of these are attributable to the common co-variation among the ASPD criteria. For readability, Table 3 provides the values. 
Table 2 Comparison of biometric models

\begin{tabular}{llllll}
\hline Model & Common factors & Specific factors & $\Delta$ df & $\Delta$ BIC & $\Delta$ SABIC \\
\hline Independent pathway models & \#A-\#C-\#E & a-c-e & & & \\
II & 1A-1C-1E & a-c-e & - & - & - \\
III & 1A-0C-1E & a-e & 14 & $\mathbf{- 8 8 . 7}$ & $\mathbf{- 4 4 . 2}^{\text {a }}$ \\
IV & 0A-1C-1E & c-e & 14 & -67.2 & -22.7 \\
V & 2A-0C-1E & a-e & 8 & -68.8 & -43.3 \\
VI & 1A-0C-2E & a-e & 8 & -63.2 & -37.8 \\
VII & 3A-0C-1E & a-e & 3 & -37.8 & -28.2 \\
Common pathway models & \#(A-C-E) & a-c-e & & & \\
II & 1-ACE & a-c-e & 12 & -43.5 & -1.28 \\
III & 1-CE & c-e & 20 & -88.7 & -21 \\
IV & 1-AE & a-e & 20 & $-\mathbf{1 0 1 . 0}$ & $-\mathbf{3 3 . 7}$ \\
V & 2-ACE & a-c-e & 0 & -7.9 & 0.22 \\
VI & 2-CE & c-e & 10 & -61.7 & -21.8 \\
VII & 2-AE & a-e & 10 & -79.5 & -39.6 \\
VIII & 3-AE & a-e & -2 & -2.8 & 3.1 \\
\hline
\end{tabular}

Numbers of factors in a biometric ACE model are given in the form A-C-E with the number of factors in front of the letters. Presence versus absence of criterion-specific effects is indicated by corresponding subset of "a-c-e" components. We denote e.g. an independent-pathway model with 2 A-factors, 0 C-factors and 1 E-factor by " $2 \mathrm{~A}-0 \mathrm{C}-1 \mathrm{E}$ ", and the presence versus absence of its specific effects by a subset "a-e" of the full variance decomposition "a-c-e". Number of factors for a common-pathway model is just a single quantity (e.g., "2-AE" for two-factor model for additive and non-shared environment, assuming shared environmental effects are negligible). Model numbering follows a previous study (Kendler et al. 2012)

Baseline values (independent-pathway Model II) were 19470 degrees of freedom (df), Bayesian Information Criterion (BIC) value of -134 855.7, and sample-size adjusted BIC (SABIC) value of 6613.2

$\Delta d f$ denotes change in model degrees of freedom compared to the baseline model, and $\triangle B I C$ denotes change in BIC. Lower BIC values indicate more parsimonious (i.e. better) model. $\triangle$ SABIC denotes change in SABIC. Lower SABIC values indicate more parsimonious (i.e. better) model

${ }^{a}$ The overall best fit according to the information criterion. Bold font indicates the overall best fit within the two subsets of models considered separately, for independent-pathway models and for common-pathway models

\section{Discussion}

To our knowledge this is the first study to fully explore the genetic and environmental factors underlying DSMIV and -5 ASPD criteria by comparing both independent and common pathway biometric models using structured interview data and a population-based sample of twins. Our main findings indicate that a single, highly heritable common factor could account for the correlations between the ASPD criteria, thereby suggesting that ASPD reflects a single shared dimension of liability, plus criterion-specific liabilities. This supports the validity of ASPD as a diagnostic construct (Franić et al. 2013), and supports the use of ASPD diagnosis in molecular genetics studies more than the previously reported two-dimensional genetic structure.

Because the content of the ASPD criteria is dispersed across several psychopathy factors (Coid and Ullrich 2010), our findings are of relevance for psychopathy research. We found that the genetic and environmental influences on multiple relevant behaviors (the ASPD criteria) are statistically associated rather than independent. This aligns with the observations that full-blown psychopathy usually involves both genetic and environmental exposures (Derefinko and Widiger 2016; Hyde et al. 2016; Viding and McCrory 2012). However, another study has reported statistically independent genetic and environmental factors, as discussed next.

Our findings differ from a previous study using twin selfreport data and examining a more restricted set of biometric models (Kendler et al. 2012). The study found evidence for two genetic factors and an independent environmental factor, plus criterion-specific genetic and environmental influences. In that study, "lack of remorse" did not load strongly onto the genetic factors and had a low overall heritability. The differences between this study and the previous study may be due to the range of models tested, the difference in the assessment formats (questionnaire versus interview) that can affect heritability estimates (Rhee and Waldman 2002), and sample differences discussed below.

First, the set of models studied by Kendler et al. (2012) did not include common pathway biometric models that would allow statistical dependence between the genetic and 


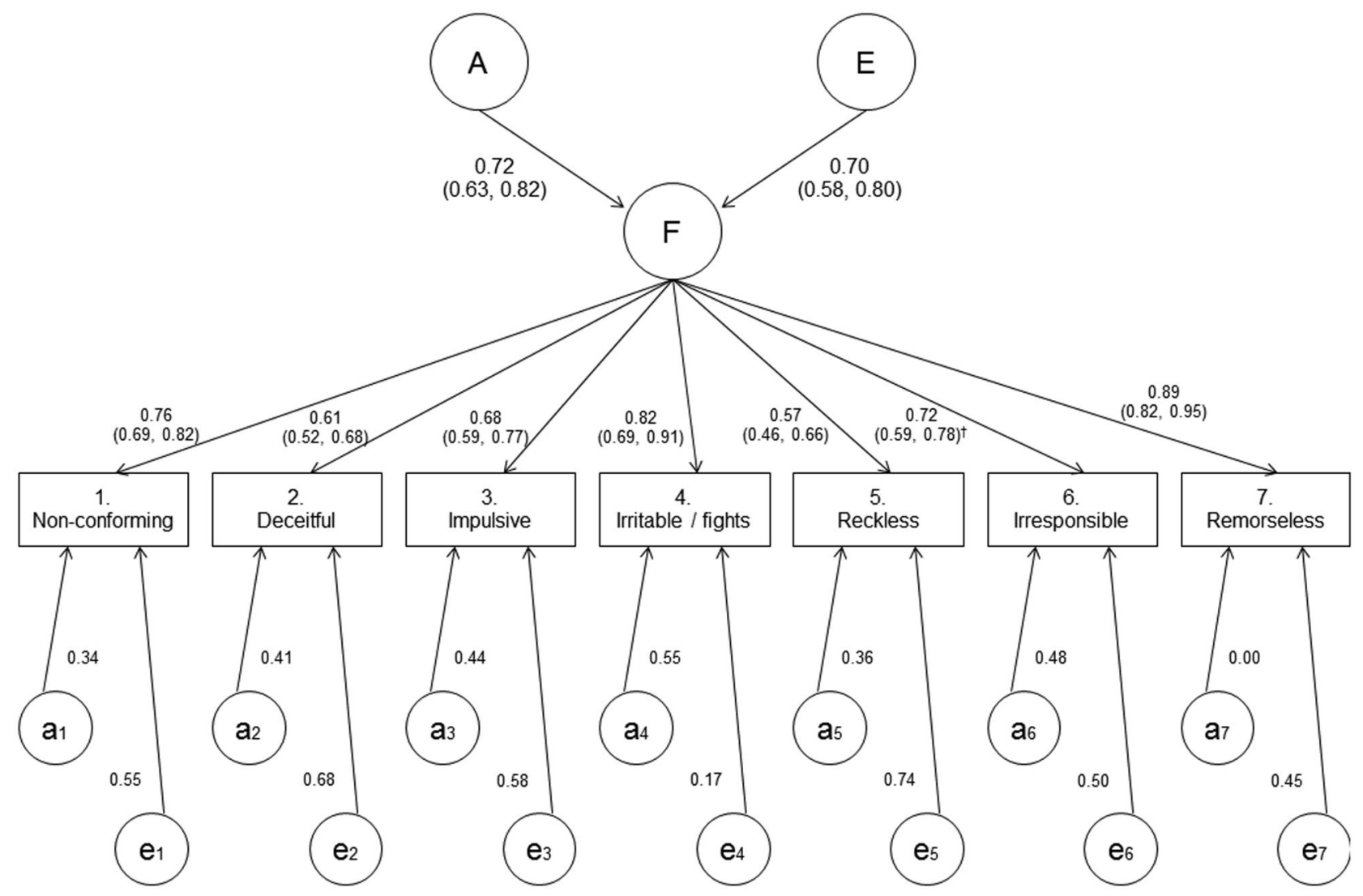

Fig. 3 Path diagram and parameter estimates of the best-fitting common-pathway biometric model. Estimates are from the model with scalar-translated rather than freely estimated threshold parameters, because that model was the best fit to data, but with all data, including separate-sex dizygotic twins. The numbers in parentheses provide 95\% likelihood-profile confidence intervals. Superscript " $\dagger$ ” refers to the one factor-loading interval estimate that did not properly converge and was estimated as the equivalent supplementary model on the

environmental influences, and therefore could not find such dependencies. Second, antisocial individuals may have weak introspective abilities. Co-twin's antisocial behavior is more accurately observed than own behavior; yet, those who are antisocial are generally less likely to perceive others as antisocial (Kendler et al. 2002). In their theoretical analysis of the effects of DIF by zygosity on estimation of heritability, Neale et al. (2005) discussed self-reported antisocial behavior as a possible case for this source of bias. Third, the genetic "aggressive-disregard" factor in the previous study reflected precisely the 3 items that have shown DIF with respect to sex in another study (Jane et al. 2007; Kendler et al. 2012). As we did not find strong indications of DIF by sex, also these differences between the samples could play a role. When multiple items show DIF with respect to the same variable (e.g., sex), the variation in this variable could show up as a common factor for the items. It would be tempting to think that an omnibus test for sex same-sex twins only (see online supplement). Furthermore, reliable confidence intervals for the specific effects were unattainable, but an omnibus test indicated that also the genetic specific effects were significant $(p=0.004)$. Squares of the path coefficients give corresponding variance proportions: e.g., the common-pathway factor explained $100 \% \times 0.89^{2}=79 \%$ of the total variance in remorselessness, or criterion 7 , of which $100 \% \times 0.72^{2}=52 \%$ is heritable variation. Table 3 lists the total heritabilities of the criteria

effects is sufficient to guard against all adverse effects of DIF, but significant findings in such a test also depend on its statistical power, whereas the detected factor number depends on another test that may or may not have comparable statistical properties. Here we did not detect DIF for multiple items with respect to the assessed variables, but assessing possible DIF for other assessment formats and/or samples might explain differences across findings.

In general, all the DSM-IV/DSM-5 ASPD criteria tend to load strongly on the same phenotypic factor (Blais and Norman 1997; Warren and South 2009; Huprich et al. 2010). Differential diagnosis has been problematic, however, since many ASPD criteria (e.g., irresponsibility, aggressiveness, impulsivity) may also be associated with other DSM-5 diagnoses, such as borderline PD, schizophrenia, bipolar disorder, or major depression (Blais and Norman 1997; Derefinko and Widiger 2016). Thus, the possibility that ASPD differs phenomenologically between 
Table 3 Total genetic and environmental variance of Antisocial Personality Disorder criteria and the percentages attributable to the common factor

\begin{tabular}{|c|c|c|c|c|}
\hline \multirow[b]{2}{*}{ Criterion } & \multicolumn{2}{|c|}{ Genetic variance } & \multicolumn{2}{|c|}{$\begin{array}{l}\text { Environmental } \\
\text { variance }\end{array}$} \\
\hline & $a^{2}$ & $\begin{array}{l}\text { Common } \\
\text { factor\% }\end{array}$ & $\mathrm{e}^{2}$ & $\begin{array}{l}\text { Com- } \\
\text { mon } \\
\text { factor\% }\end{array}$ \\
\hline 1. Not conforming & 0.41 & 74 & 0.59 & 48 \\
\hline 2. Deceitfulness & 0.36 & 54 & 0.64 & 28 \\
\hline 3. Impulsivity, or failure to plan & 0.44 & 57 & 0.56 & 41 \\
\hline 4. Irritability/repeated fights & 0.67 & 53 & 0.33 & 100 \\
\hline 5. Reckless disregard & 0.28 & 61 & 0.72 & 22 \\
\hline 6. Irresponsibility & 0.49 & 56 & 0.51 & 49 \\
\hline 7. Lack of remorse & 0.42 & 100 & 0.58 & 66 \\
\hline
\end{tabular}

The values correspond the best-fit model illustrated in the Fig. 3 . "Common factor\%" refers to the percentage of the variance component that is attributable to the biometric common-pathway factor, the rest being attributable to the influences specific to the given criterion

$a^{2}$ heritability or proportion of variance in liability to endorse a criterion because of genetic factors, $e^{2}$ proportion of variance in liability attributable to environmental factors

individuals who satisfy versus do not satisfy a specific characteristic criterion, such as "lack of remorse", has been investigated (Goldstein et al. 2006). Even among those who fulfilled the ASPD diagnostic criteria, lack of remorse was associated with violent behaviors. Among the other criteria, especially criterion \#4 ("irritable/fights") was associated with lack of remorse in those who obtained the ASPD diagnosis. Nevertheless, the authors concluded that there was no sufficient evidence to propose that lack of remorse would characterize a subtype of ASPD, instead suggesting "multivariate analytic approaches to examining phenomenologic heterogeneity within the ASPD diagnosis" as a "potentially fruitful avenue for future investigations" (Goldstein et al. 2006). This study represents one such analysis. Our main findings align with these previous phenotypic observations in the sense that we found a clear main factor (no subtypes), with "irritability/aggression" and "lack of remorse" as its strongest representatives.

However, the specific genetic effects we found also imply that some people who are relatively low on the latent ASPD trait can nevertheless have a stable tendency to fulfill the diagnostic criteria. Although the genetic 'residual' liabilities are uncorrelated in the population, some individuals by chance end up having multiple residual contributions. While the specific effects in classic EFA are frequently interpreted as (unstable) measurement errors, such an interpretation does not carry over to specific genetic effects in biometric factor analysis because the measurement errors are already contained in the environmental specific effects. This means that simple aggregates of ASPD criteria, such as sum scores or DSM-IV and DSM-5 diagnoses, will contain both genetic influences related to overall ASPD and genetic influences unrelated to overall ASPD (i.e., influences specific to a single criterion).

Our findings have implications for nosology (Livesley 2005; Kendler 2006), psychometrics (Livesley 2005; Franić et al. 2013), molecular genetics (Tielbeek et al. 2012; Salvatore et al. 2015), developmental psychopathology (Hyde et al. 2016; Viding and McCory 2012), and human behavioral ecology and evolution (Nettle et al. 2013; Ribeiro da Silva et al. 2015; Del Giudice et al. 2011; Colman and Wilson 1997; Ellis 1988). Our study is relevant to all these research fields in providing evidence that a unidimensional ASPD phenotype exists and permeates the domains of genetic and environmental influences. Suggestive of robustness in findings, ASPD and borderline personality share much of their heritability (Kendler et al. 2008; Torgersen et al. 2008; Reichborn-Kjennerud et al. 2015), and a biometric analysis found a common pathway model superior to the tested independent pathway models also in the case of borderline personality disorder (Reichborn-Kjennerud et al. 2013). Furthermore, theoretical arguments distinguish these two personality disorders from other personality constructs that have not shown unambiguous common pathway structure (Brüne 2016; Ribeiro da Silva et al. 2015; Franić et al. 2014). Nevertheless, the findings reported here should be interpreted in light of the following limitations.

\section{Limitations}

Finding that a single-factor common pathway model fit the multivariate data for the DSM-IV ASPD criteria best is consistent with the notion of a single mechanism that generates variation along a single dimension, but not a sufficient condition to exclude all other possible explanations. For example, it has been shown by means of theoretical analysis that a causal process of "mutualism" may generate data that fits well with models of underlying latent cause even if there is not one (van der Maas et al. 2006). Dynamic developmental cascades among 'criteria' might create correlation structures that give a false impression of a latent factor. This could also confound genetic correlations if the triggering criteria are partly heritable, causing the entire developmental cascade to reflect the same heritable triggers. However, the most salient indicator item in the common pathway model estimated in this study was "lack of remorse", which appears consistent with a biological mode of strategic behavior that emphasizes exploitation over cooperation (Del Giudice et al. 2011; Ribeiro da Silva et al. 2015).

Another possible limitation is that the attrition in our sample could have had an effect on the structural estimates. In a previous study, nonparticipation in the sample 
was predicted by dizygosity, male sex, being married, having children, lower education, and few indicators of poor mental and somatic health and unhealthy lifestyle (Tambs et al. 2009). However, one might generally expect attrition to introduce complex dependencies and thereby weaken rather than strengthen the evidence for a single-factor common pathway structure. Thus, the moderate attrition effects (Tambs et al. 2009) appear to be an unlikely source of bias for our main findings. As a general limitation applicable to all related studies, optimal information criterion for purposes of model selection is still a debated topic (Markon and Krueger 2004; Nylund et al. 2007; Vrieze 2012; Bulteel et al. 2013), and even in the cases where BIC indicated very strong support for a model, SABIC did not always do so (Table 2 and Table S3).

This study was limited to ASPD criteria and the extent to which the common pathway etiology of the ASPD criteria corresponds to the biometric structure of psychopathy remains an open question (Derefinko and Widiger 2016; Wygant et al. 2016). Moreover, a full diagnosis of ASPD requires a presence of conduct disorder before the age of 15 years, and analytic treatment of conduct disorder varies across studies (Jane et al. 2007; Kendler et al. 2012, 2013). However, in the online supplement we present results showing that its inclusion made relatively little difference here. Due to statistical-power considerations, we did not explicitly study genetic sex-limitation in the sense of assessing whether male and female ASPD could be associated with distinct pools of genes (Neale et al. 2006), but previous studies have not found such differences in adolescent or adult antisocial behavior (Jacobson et al. 2002; Larsson et al. 2006). Overall, only few people in our sample exceed the usual diagnostic thresholds and the generalizability of the results is therefore dependent on the dimensional nature of the studied phenomena (Marcus et al. 2006).

When there are unaccounted clustering (dependencies) in data, likelihood-based statistics are not necessarily reliable (Pornprasertmanit et al. 2014). We modeled twindependencies similarly to the previous study (Kendler et al. 2012), but neither those nor our phenotypic factor analyses explicitly took into account the different phenotypic dependence structures across MZ and DZ twins. Nevertheless, both the studies obtained highly consistent phenotypic and biometric results, albeit different ones.

Acknowledgements We acknowledge funding from the US National Institutes of Health and National Institute on Drug Abuse (1R01DA037558-01A1), the Research Council of Norway (226985), the Norwegian Foundation for Health and Rehabilitation, the Norwegian Council for Mental Health, and the European Commission under the program "Quality of Life and Management of the Living Resources" of the Fifth Framework Program (QLG2CT-2002-01254). THR had full access to all the data in this study and takes responsibility for the integrity of the data and the accuracy of the data analysis. The funding sources had no role in the design and conduct of the study; collection, management, analysis, and interpretation of the data; preparation, review, or approval of the manuscript; and decision to submit the manuscript for publication.

\section{Compliance with Ethical Standards}

Conflict of interest Tom Rosenström, Eivind Ystrom, Fartein Ask Torvik, Nikolai Olavi Czajkowski, Nathan A. Gillespie, Steven H. Aggen, Robert F. Krueger, Kenneth S. Kendler, Ted Reichborn-Kjennerud declare that they have no conflict of interest.

Ethical Approval Approval was received from The Norwegian Data Inspectorate and the Regional Committee for Medical and Health Research Ethics, and written informed consent was obtained from all participants after a complete description of the study.

Human and Animal Rights All procedures performed in studies involving human participants were in accordance with the ethical standards of the institutional and/or national research committee and with the 1964 Helsinki declaration and its later amendments or comparable ethical standards.

Informed Consent Informed consent was obtained from all individual participants included in the study.

\section{References}

American Psychiatric Association (2013) Diagnostic and statistical manual of mental disorders, Fifth Edition. American Psychiatric Association, Arlington

Asparouhov $\mathrm{T}$ (2005) Sampling weights in latent variable modeling. Struct Equ Model 12:411-434. doi:10.1207/ s15328007sem1203_4

Blais MA, Norman DK (1997) A psychometric evaluation of the DSM-IV personality disorder criteria. J Personal Disord 11:168176. doi:10.1521/pedi.1997.11.2.168

Boker S, Neale M, Maes H et al (2011) OpenMx: an open source extended structural equation modeling framework. Psychometrika 76:306-317. doi:10.1007/s11336-010-9200-6

Brüne M (2016) Borderline personality disorder: why "fast and furious"? Evol Med Public Health. doi:10.1093/emph/eow002

Bulteel K, Wilderjans TF, Tuerlinckx F, Ceulemans E (2013) CHull as an alternative to AIC and BIC in the context of mixtures of factor analyzers. Behav Res Methods 45:782-791. doi:10.3758/ s13428-012-0293-y

Choi SW, Gibbons LE, Crane PK (2011) lordif: An R package for detecting differential item functioning using iterative hybrid ordinal logistic regression/item response theory and Monte Carlo simulations. J Stat Softw 39:1-30. doi:10.18637/jss.v039.i08

Coid J, Ulrich S (2010) Antisocial personality disorder is on a continuum with psychopathy. Compr Psychiatry 51:426-433. doi:10.1016/j.comppsych.2009.09.006

Colman AM \& Wilson JC (1997) Antisocial personality disorder: an evolutionary game theory analysis. Legal Criminol Psychol 2:23-34.

Del Giudice M, Ellis BJ, Shirtcliff EA (2011) The adaptive calibration model of stress responsivity. Neurosci Biobehav Rev 35:15621592. doi:10.1016/j.neubiorev.2010.11.007

Derefinko KJ, Widiger TA (2016) Antisocial personality disorder. In: Fatemi SH, Clayton PJ (Eds.), The medical basis of psychiatry, 4th edn. Springer, New York, pp 229-245 
Ellis L (1988) Criminal behavior and $\mathrm{r} / \mathrm{K}$ selection: An extension of gene-based evolutionary theory. Person Individ Diff 9:697-708. doi:10.1016/0191-8869(88)90059-1

Ficks CA, Waldman ID (2014) Candidate genes for aggression and antisocial behavior: a meta-analysis of association studies of the 5HTTLPR and MAOA-uVNTR. Behav Genet 44:427-444. doi:10.1007/s10519-014-9661-y

Franić S, Dolan CV, Borsboom D et al (2013) Can genetics help psychometrics? Improving dimensionality assessment through genetic factor modeling. Psychol Methods 18:406-433. doi:10.1037/a0032755

Franić S, Borsboom D, Dolan CV, Boomsma DI (2014) The big five personality traits: psychological entities or statistical constructs? Behav Genet 44:591-604. doi:10.1007/s10519-013-9625-7

Goldstein RB, Grant BF, Huang B, Smith SM, Stinson FS, Dawson DA, Chou SP (2006) Lack of remorse in antisocial personality disorder: sociodemographic correlates, symptomatic presentation, and comorbidity with Axis I and Axis II disorders in the National Epidemiologic Survey on Alcohol and Related Conditions. Comp Psychiatry 47:289-297. doi:10.1016/j. comppsych.2005.11.002

Good P (2005) Permutation, parametric, and bootstrap tests of hypotheses, 3rd edn. Springer, New York

Harris JR, Magnus P, Tambs K (2002) The Norwegian Institute of Public Health Twin Panel: a description of the sample and program of research. Twin Res 5:415-423. doi:10.1375/136905202320906192

Hayashi K, Bentler PM, Yuan K-H (2007) On the likelihood ratio test for the number of factors in exploratory factor analysis. Struct Equ Model 14:505-526. doi:10.1080/10705510701301891

Heath AC, Eaves LJ, Martin NG (1989) The genetic structure of personality III. Multivariate genetic item analysis of the EPQ scales. Personal Individ Differ 10:877-888. doi:10.1016/0191-8869(89)90023-8

Helgeland MI, Kjelsberg E, Torgersen S (2005) Continuities between emotional and disruptive behavior disorders in adolescence and personality disorders in adulthood. Am J Psychiatry 162:19411947. doi:10.1176/appi.ajp.162.10.1941

Horn JL (1965) A rationale and test for the number of factors in factor analysis. Psychometrika 30:179-185

Humphreys LG, Montanelli RG Jr (1975) An investigation of the parallel analysis criterion for determining the number of common factors. Multivar Behav Res 10:193-205

Huprich SK, Schmitt TA, Richard DCS, et al (2010) Comparing factor analytic models of the DSM-IV personality disorders. Personal Disord 1:22-37. doi:10.1037/a0018245

Hyde LW, Waller R, Trentacosta CJ, Shaw DS, Neiderhiser JM, Ganiban JM, Reiss D, Leve LD (2016) Heritable and nonheritable pathways to early callous-unemotional behaviors. Am J Psychiatry 173:903-910. doi:10.1176/appi.ajp.2016.15111381

Jacobson KC, Prescott CA, Kendler KS (2002) Sex differences in the genetic and environmental influences on the development of antisocial behavior. Dev Psychopathol 14:395-416. doi:10.1017/ S0954579402002110

Jane JS, Oltmanns TF, South SC, Turkheimer E (2007) Gender bias in diagnostic criteria for personality disorders: an item response theory analysis. J Abnorm Psychol 116:166-175. doi:10.1037/0021-843X.116.1.166

Jang KL, Livesley WJ, Angleitner A, et al (2002) Genetic and environmental influences on the covariance of facets defining the domains of the five-factor model of personality. Personal Individ Differ 33:83-101. doi:10.1016/S0191-8869(01)00137-4

Johnson W, Krueger RF (2004) Genetic and environmental structure of adjectives describing the domains of the Big Five Model of personality: A nationwide US twin study. J Res Personal 38:448472. doi:10.1016/j.jrp.2003.11.001
Jones RH (2011) Bayesian information criterion for longitudinal and clustered data. Stat Med 30:3050-3056. doi:10.1002/sim.4323

Kass RE, Raftery AE (1995) Bayes factors. J Am Stat Assoc 90:773795. doi:10.1080/01621459.1995.10476572

Kendler KS (2006) Reflections on the relationship between psychiatric genetics and psychiatric nosology. Am J Psychiatry 163:1138-1146. doi:10.1176/ajp.2006.163.7.1138

Kendler KS, Silberg JL, Neale MC et al (1992) Genetic and environmental factors in the aetiology of menstrual, premenstrual and neurotic symptoms: a population-based twin study. Psychol Med 22:85-100. doi:10.1017/S0033291700032761

Kendler KS, Prescott CA, Myers J, Neale MC (2002) The joint analysis of personal interview and family history diagnoses: evidence for validity of diagnosis and increased heritability estimates. Psychol Med 32:829-842. doi:10.1017/S0033291702005858

Kendler KS, Aggen SH, Czajkowski N et al (2008) The structure of genetic and environmental risk factors for DSM-IV personality disorders: a multivariate twin study. Arch Gen Psychiatry 65:1438-1446. doi:10.1001/archpsyc.65.12.1438

Kendler KS, Aggen SH, Patrick CJ (2012) A multivariate twin study of the DSM-IV criteria for antisocial personality disorder. Biol Psychiatry 71:247-253. doi:10.1016/j.biopsych.2011.05.019

Kendler KS, Aggen SH, Patrick CJ (2013) Familial influences on conduct disorder reflect 2 genetic factors and 1 shared environmental factor. JAMA Psychiatry 70:78-86. doi:10.1001/ jamapsychiatry.2013.267

Larsson H, Andershed H, Lichtenstein P (2006) A genetic factor explains most of the variation in the psychopathic personality. J Abnorm Psychol 115:221-230. doi:10.1037/0021-843X.115.2.221

Larsson H, Tuvblad C, Rijsdijk FV et al (2007) A common genetic factor explains the association between psychopathic personality and antisocial behavior. Psychol Med 37:15-26. doi:10.1017/ S003329170600907X

Lawley DN, Maxwell AE (1971) Factor Analysis as a Statistical Method., 2nd edn. Butterworths \& Co, London

Livesley WJ (2005) Behavioral and molecular genetic contributions to a dimensional classification of personality disorder. J Personal Disord 19:131-155. doi:10.1521/pedi.19.2.131.62631

MacIntosh R, Hashim S (2003) Variance estimation for converting MIMIC model parameters to IRT parameters in DIF analysis. Appl Psychol Meas 27:372-379. doi: $10.1177 / 0146621603256021$

Marcus DK, Lilienfeld SO, Edens JF, Poythress NG (2006) Is antisocial personality continuous or categorical? A taxometric analysis. Psychol Med 36:1571-1581. doi:10.1017/S0033291706008245

Markon KE, Krueger RF (2004) An empirical comparison of information-theoretic selection criteria for multivariate behavior genetic models. Behav Genet 34:593-610. doi:10.1007/ s10519-004-5587-0

Muthén BO (1989) Using item-specific instructional information in achievement modeling. Psychometrika 54:385-396. doi:10.1007/ BF02294624

Muthén BO, Kao C-F, Burstein L (1991) Instructionally sensitive psychometrics: application of a new IRT-based detection technique to mathematics achievement test items. J Educ Meas 28:1-22. doi:10.1111/j.1745-3984.1991.tb00340.x

Neale MC (2003) A finite mixture distribution model for data collected from twins. Twin Res 6:235-239. doi: $10.1375 / 136905203765693898$

Neale MC, Maes HHM (2002) Methodology for genetic studies of twins and families (an online version revised from Neale MC \& Cardon L, 1992, Methodology for genetic studies of twins and families. Kluwer Academic, Dordrecht, the Netherlands). http://ibgwww.colorado.edu/twins2003/cdrom/HTML/BOOK/ book2002c.pdf 
Neale MC, Miller MB (1997) The use of likelihood-based confidence intervals in genetic models. Behav Genet 27:113-120

Neale MC, Lubke G, Aggen SH, Dolan CV (2005) Problems with using sum scores for estimating variance components: contamination and measurement noninvariance. Twin Res Hum Genet Off J Int Soc Twin Stud 8:553-568. doi:10.1375/183242705774860231

Neale MC, Røysamb E, Jacobson K (2006) Multivariate genetic analysis of sex limitation and G x E interaction. Twin Res Hum Genet 9:481-489. doi:10.1375/183242706778024937

Nettle D, Gibson MA, Lawson DW, Sear R (2013) Human behavioral ecology: current research and future prospects. Behav Ecol 24:1031-1040. doi:10.1093/beheco/ars222

Neumann GS, Hare RD, Pardini DA (2015) Antisociality and the construct of psychopathy: Data from across the globe. J Pers 86:678-692. doi:10.1111/jopy. 12127

Nylund KL, Asparouhov T, Muthén BO (2007) Deciding on the number of classes in latent class analysis and growth mixture modeling: a monte carlo simulation study. Struct Equ Model 14:535569. doi:10.1080/10705510701575396

Pappa I, St Pourcain B, Benke K et al (2016) A genome-wide approach to children's aggressive behavior: the EAGLE consortium. Am J Med Genet B Neuropsychiatr Genet 171:562-572. doi:10.1002/ajmg.b.32333

Penfield RD, Camilli G (2007) Differential item functioning and item bias. In: Rao CR, Sinharay S (eds) Handbook of statistics, vol 26: Psychometrics. Noth-Holland, Amsterdam, pp 125-167

Pfohl B, Blum N, Zimmerman M (1995) Structured interview for DSM-IV personality (SIDP-IV). University of Iowa, Department of Psychiatry, Iowa

Plomin R, DeFries JC, Knopik VS, Neiderheiser J (2012) Behavioral Genetics, 6th edn. Worth Publishers, New York

Pornprasertmanit S, Lee J, Preacher KJ (2014) Ignoring clustering in confirmatory factor analysis: Some consequences for model fit and standardized parameter estimates. Multivariate Behav Res 49:518-543. doi:10.1080/00273171.2014.933762

Reichborn-Kjennerud T, Ystrom E, Neale MC et al (2013) Structure of genetic and environmental risk factors for symptoms of DSMIV Borderline Personality Disorder. JAMA Psychiatry 70:12061214. doi:10.1001/jamapsychiatry.2013.1944

Reichborn-Kjennerud T, Czajkowski N, Ystrøm E et al (2015) A longitudinal twin study of borderline and antisocial personality disorder traits in early to middle adulthood. Psychol Med 45:31213131. doi: $10.1017 /$ S0033291715001117

Rhee SH, Waldman ID (2002) Genetic and environmental influences on antisocial behavior: a meta-analysis of twin and adoption studies. Psychol Bull 128:490-529. doi:10.1037//0033-2909.128.3.490

Ribeiro da Silva D, Rijo D, Salekin RT (2015) The evolutionary roots of psychopathy. Aggress Violent Behav 21:85-96. doi:10.1016/j. avb.2015.01.006
Salvatore JE, Edwards AC, McClintick JN et al (2015) Genome-wide association data suggest $\mathrm{ABCB} 1$ and immune-related gene sets may be involved in adult antisocial behavior. Trans Psychiatry 5:e558. doi:10.1038/tp.2015.36

Sclove SL (1987) Application of model-selection criteria to some problems in multivariate analysis. Psychometrika 52:333-343. doi:10.1007/BF02294360

Tambs K, Rønning T, Prescott CA et al (2009) The Norwegian Institute of Public Health twin study of mental health: examining recruitment and attrition bias. Twin Res Hum Genet 12:158-168. doi:10.1375/twin.12.2.158

Tielbeek JJ, Medland SE, Benyamin B et al (2012) Unraveling the genetic etiology of adult antisocial behavior: a genome-wide association study. PLoS One 7:e45086. doi:10.1371/journal. pone. 0045086

Torgersen S, Kringlen E, Cramer V (2001) The prevalence of personality disorders in a community sample. Arch Gen Psychiatry 58:590-596. doi:10.1001/archpsyc.58.6.590

Torgersen S, Czajkowski N, Jacobson K et al (2008) Dimensional representations of DSM-IV cluster B personality disorders in a population-based sample of Norwegian twins: a multivariate study. Psychol Med 38:1617-1625. doi:10.1017/S0033291708002924

Torgersen S, Myers J, Reichborn-Kjennerud T, Røysamb E, Kubarych TS, Kendler KS (2012) The heritability of Cluster B personality disorders assessed both by personal interview and questionnaire. J Personal Disord 26:848-866. doi:10.1521/pedi.2012.26.6.848

van der Maas HLJ, Dolan CV, Grasman RPPP et al (2006) A dynamical model of general intelligence: the positive manifold of intelligence by mutualism. Psychol Rev 113:842-861

Venables NC, Hall JR, Patrick CJ (2014) Differentiating psychopathy from antisocial personality disorder: a triarchic model perspective. Psychol Med 44:1005-1013. doi:10.1017/ S003329171300161X

Viding E, McCrory EJ (2012) Genetic and neurocognitive contributions to the development of psychopathy. Dev Psychopathol 24:969-983. doi:10.1017/S095457941200048X

Vrieze SI (2012) Model selection and psychological theory: a discussion of the differences between the Akaike information criterion (AIC) and the Bayesian information criterion (BIC). Psychol Methods 17:228-243. doi:10.1037/a0027127

Warren JI, South SC (2009) A symptom level examination of the relationship between Cluster B personality disorders and patterns of criminality and violence in women. Int J Law Psychiatry 32:10 17. doi:10.1016/j.ijlp.2008.11.005

Wygant DB, Sellbom M, Sleep CE, et al (2016) Examining the DSM-5 alternative personality disorder model operationalization of antisocial personality disorder and psychopathy in a male correctional sample. Personal Disord. doi:10.1037/per0000179 